\title{
Hydration of a Binding Site with Restricted Solvent Access - Solvatochromic Shift of the Electronic Spectrum of a Ruthenium Polypyridine Complex, One Molecule at a Time - Supporting Information
}

\author{
Shuang Xu, ${ }^{a}$ James E. T. Smith ${ }^{b}$ and J. Mathias Weber ${ }^{b 1}$ \\ ${ }^{a}$ JILA and Department of Physics, University of Colorado, 440 UCB, \\ Boulder, CO 80309-0440, USA \\ ${ }^{b} J I L A$ and Department of Chemistry and Biochemistry, University of Colorado, 440 UCB, \\ Boulder, CO 80309-0440, USA
}

\section{Table of Contents}

Figure S1. Calculated minimum energy structures of $\left[\mathrm{RuBT}^{-\mathrm{OH}_{2}}\right]^{2+} \cdot\left(\mathrm{H}_{2} \mathrm{O}\right)_{n}$ for $n=0-4 . \quad$ S2

Figure S2. Mass spectrum of $\left[\mathrm{RuBT}-\mathrm{OH}_{2}\right]^{2+} \cdot\left(\mathrm{H}_{2} \mathrm{O}\right)_{n}$ cluster ions and other species in the same mass range.

Figure S3. Action and depletion spectra of $\left[\mathrm{RuBT}-\mathrm{OH}_{2}\right]^{2+}$ at $180 \mathrm{~K}$.

Figure S4. Photodissociation spectra for $\left[\mathrm{RuBT}-\mathrm{OH}_{2}\right]^{2+} \cdot \mathrm{H}_{2} \mathrm{O}$.

Figure S5. Photodissociation spectra for $\left[\mathrm{RuBT}-\mathrm{OH}_{2}\right]^{2+} \cdot\left(\mathrm{H}_{2} \mathrm{O}\right)_{2}$.

Procedures for determining the MLCT band envelope peak positions and centers of gravity (Figure S6)

Figure S8. Photodissociation spectrum of $\left[\mathrm{RuBT}-\mathrm{OH}_{2}\right]^{2+} \cdot\left(\mathrm{H}_{2} \mathrm{O}\right)_{2}$ and TDDFT Spectra for $[\mathrm{RuBT}-\mathrm{OH}]^{2+}$ and $[\mathrm{RuBT}-\mathrm{OH}]^{+}$

${ }^{1}$ Corresponding author; email: weberjm@jila.colorado.edu 


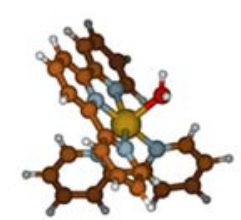

0

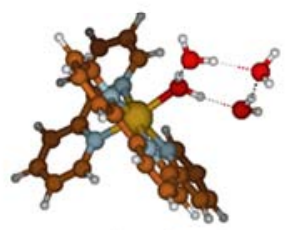

3-A $(0 \mathrm{~kJ} / \mathrm{mol})$

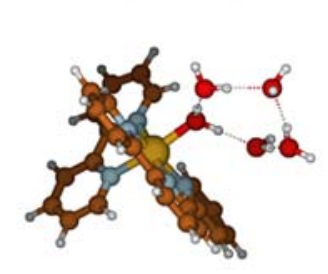

$4-\mathrm{A}(0 \mathrm{~kJ} / \mathrm{mol})$

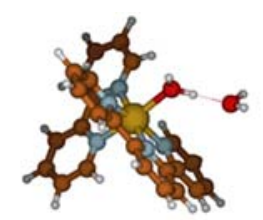

1

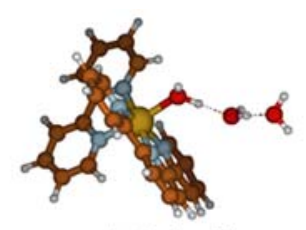

$2-\mathrm{A}(0 \mathrm{~kJ} / \mathrm{mol})$

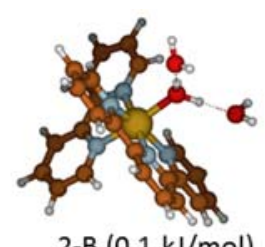

2-B $(0.1 \mathrm{~kJ} / \mathrm{mol})$

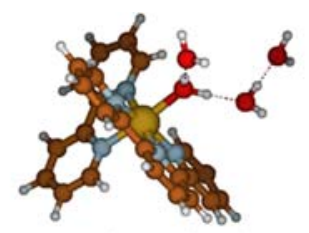

3-B $(4.4 \mathrm{~kJ} / \mathrm{mol})$

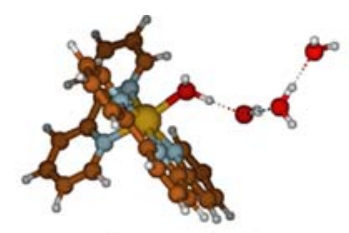

$3-\mathrm{C}(5.5 \mathrm{~kJ} / \mathrm{mol})$

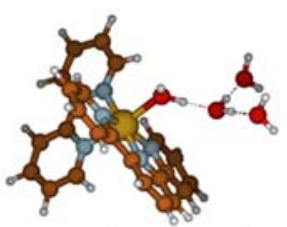

$3-D(7.8 \mathrm{~kJ} / \mathrm{mol})$

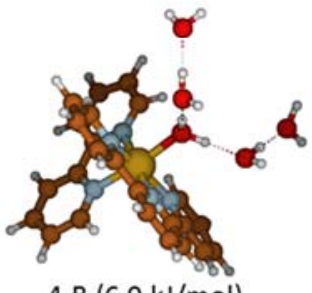

4-B $(6.9 \mathrm{~kJ} / \mathrm{mol})$

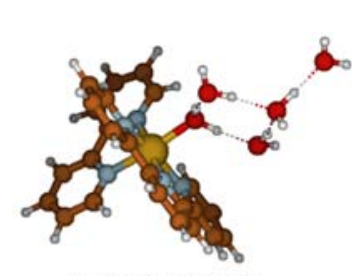

4-C $(9.3 \mathrm{~kJ} / \mathrm{mol})$

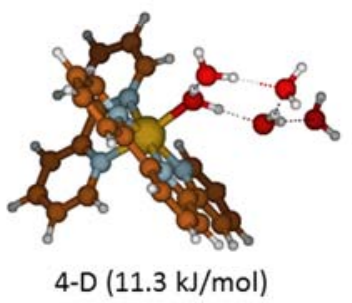

Figure S1. Calculated structures of $\left[\mathrm{RuBT}-\mathrm{OH}_{2}\right]^{2+} \cdot\left(\mathrm{H}_{2} \mathrm{O}\right)_{n}$ for $n=0-4$. Energy relative to the minimum energy structure is noted in parentheses. 


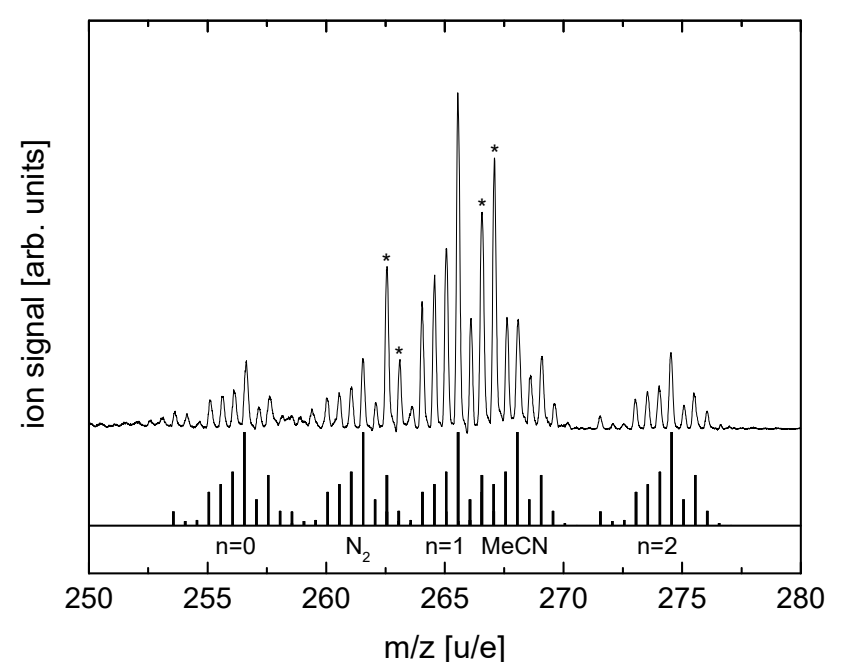

Figure S2. Mass spectrum of $\left[\mathrm{RuBT}-\mathrm{OH}_{2}\right]^{2+} \cdot\left(\mathrm{H}_{2} \mathrm{O}\right)_{n}$ cluster ions and other species in the same mass range. The settings for this particular mass spectrum were optimized for formation of the complex with $n=2$. The top trace is an experimental mass spectrum, the bottom trace shows a simulated mass spectrum for the species with $n=0-2$, together with the dicationic complexes of $\mathrm{RuBT}^{2+}$ with $\mathrm{N}_{2}$, $\left[\mathrm{RuBT}-\mathrm{N}_{2}\right]^{2+}$, and with acetonitrile, $\left[\mathrm{RuBT}-\mathrm{NCCH}_{3}\right]^{2+}$. Asterisks mark contributions from unidentified species. Note that the intensity distribution for the two largest isotopologues for $n=1$ is within $2 \%$ of the predicted isotopic abundance, so the unidentified ions in this region are not expected to contribute to the spectrum of $n=1$. 


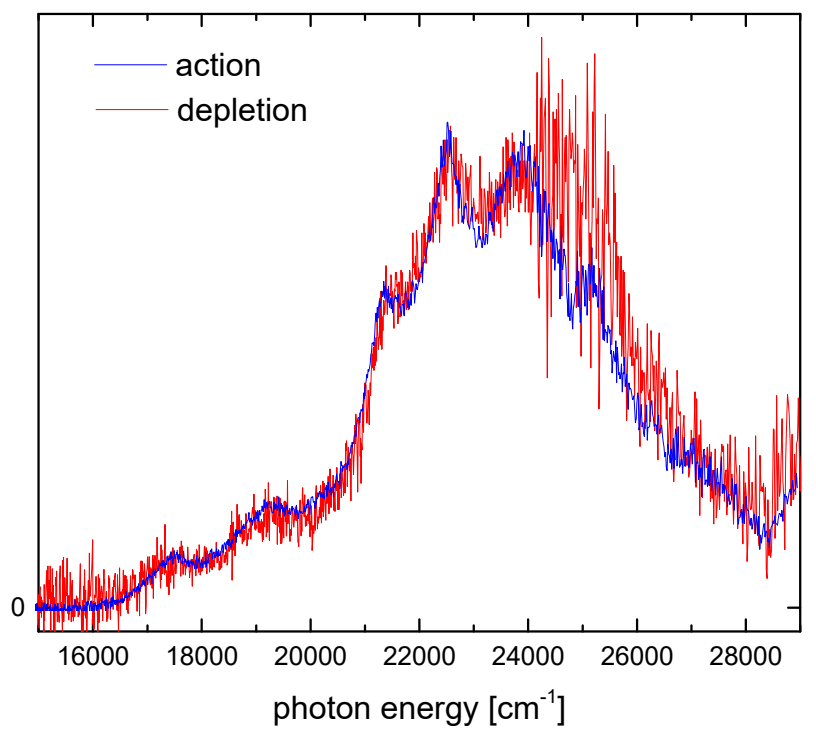

Figure S3. Action (blue) and depletion (red) spectra of $\left[\mathrm{RuBT}-\mathrm{OH}_{2}\right]^{2+}$ at $180 \mathrm{~K}$. Increased noise on the high-energy slope is due to the relatively low laser power in this spectral region. 


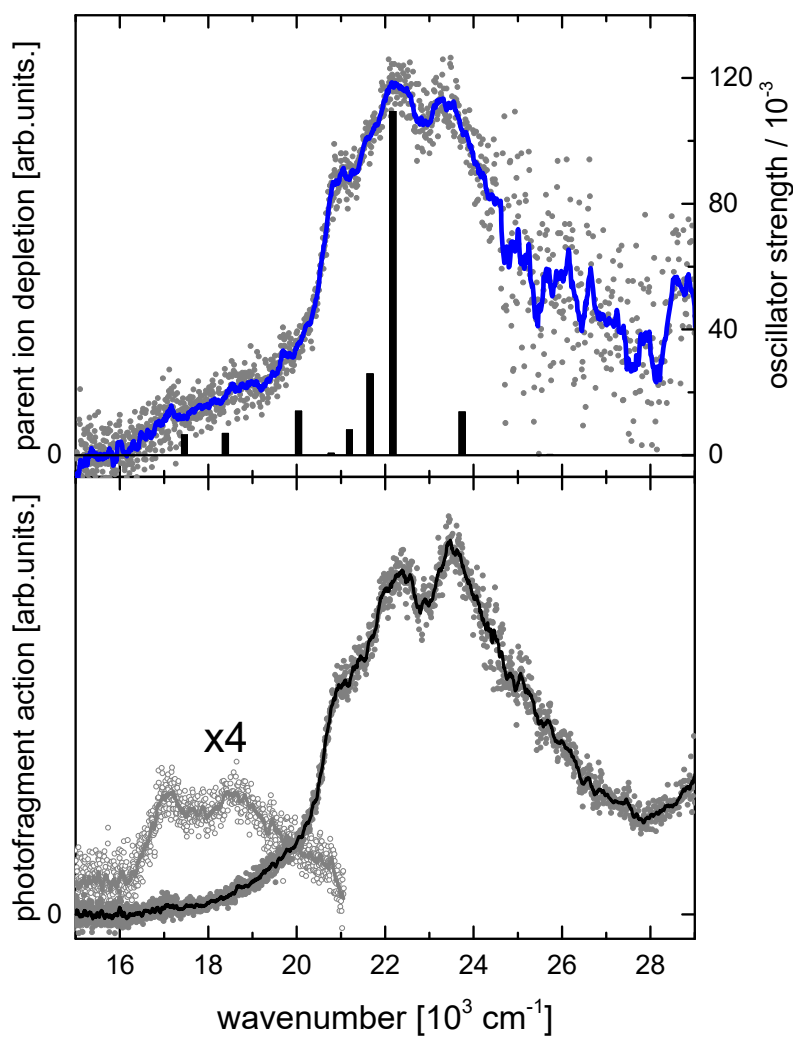

Figure S4. Photodissociation spectra for $\left[\mathrm{RuBT}-\mathrm{OH}_{2}\right]^{2+} \cdot \mathrm{H}_{2} \mathrm{O}$. Top panel: photodepletion spectrum and calculated vertical excitation spectrum (B3LYP\def2-TZVP, black columns), shifted by -1210 $\mathrm{cm}^{-1}(-0.15 \mathrm{eV})$. Bottom panel: photodissociation action traces for loss of a single water molecule (open circles, grey full line) and for the loss of two water molecules (filled circles, black full line). Circles are raw data points, the full lines are 15-point adjacent averages. The fragment action spectra show a pronounced energy dependence of the fragment formation efficiencies. The low energy onset of the spectrum is dominated by the loss of a single water molecule (presumably the solvent, calculated threshold energy $57.9 \mathrm{~kJ} / \mathrm{mol}(0.60 \mathrm{eV})$, and the loss of two water molecules while detectable - is somewhat suppressed. At ca. $19400 \mathrm{~cm}^{-1}(2.40 \mathrm{eV})$, the loss of both the solvent molecule and the aqua ligand (calculated threshold energy $147.6 \mathrm{~kJ} / \mathrm{mol}(1.53 \mathrm{eV})$ takes over and dominates the shape of the spectrum. This can be explained by interpreting the loss of the water molecules as a sequential process, with an energy-dependent change in survival probability of the $\left[\mathrm{RuBT}-\mathrm{OH}_{2}\right]^{2+}$ intermediate fragment ion (i.e., after the solvent molecule has been lost). With sufficient energy input, this ion will also lose the aqua ligand, leading to the observed increase of the branching ratio into in the $\mathrm{RuBT}^{2+}$ fragment channel for higher photon energies. 


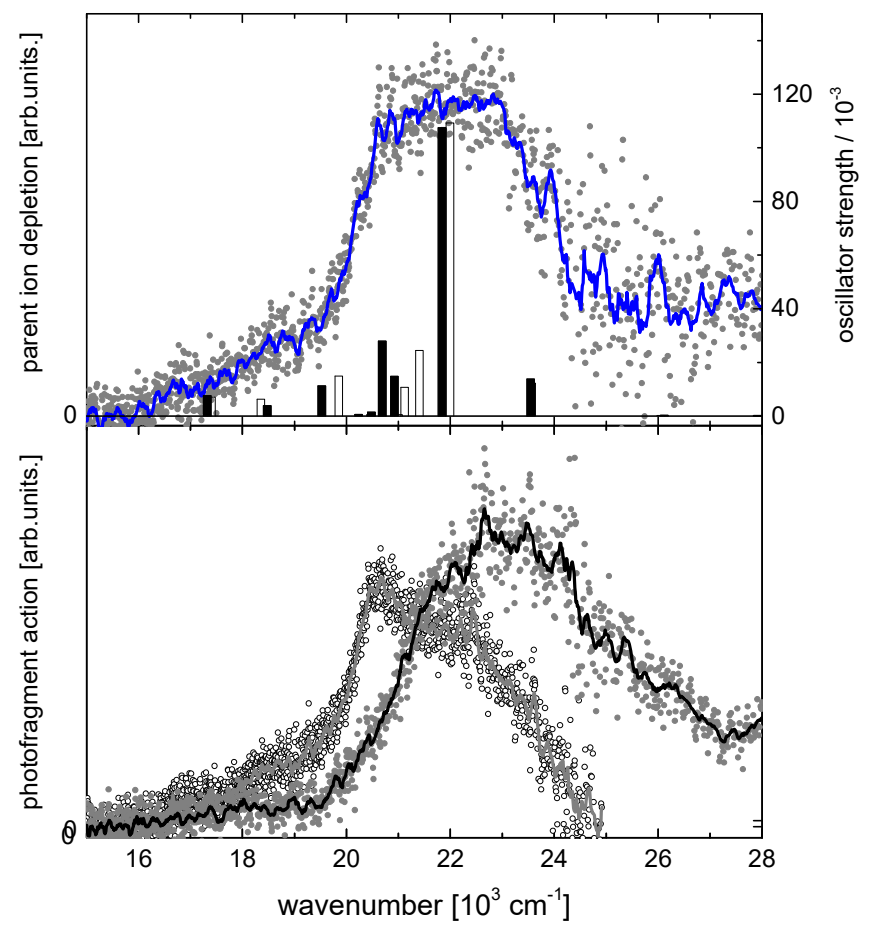

Figure S5. Photodissociation spectra for $\left[\mathrm{RuBT}-\mathrm{OH}_{2}\right]^{2+} \cdot\left(\mathrm{H}_{2} \mathrm{O}\right)_{2}$. Top panel: photodepletion spectrum and calculated vertical excitation spectrum (columns), shifted by $-1210 \mathrm{~cm}^{-1}(-0.15 \mathrm{eV})$. The open columns represent the 2-A isomer, the filled columns show the 2-B isomer. Bottom panel: photodissociation action traces for loss of two water molecules (open circles, grey full line) and for the loss of all three water molecules (filled circles, black full line). Circles are raw data points, the full lines are 15-point adjacent averages. 


\section{Procedures for the determination of MLCT band envelope peak positions and center of gravity positions}

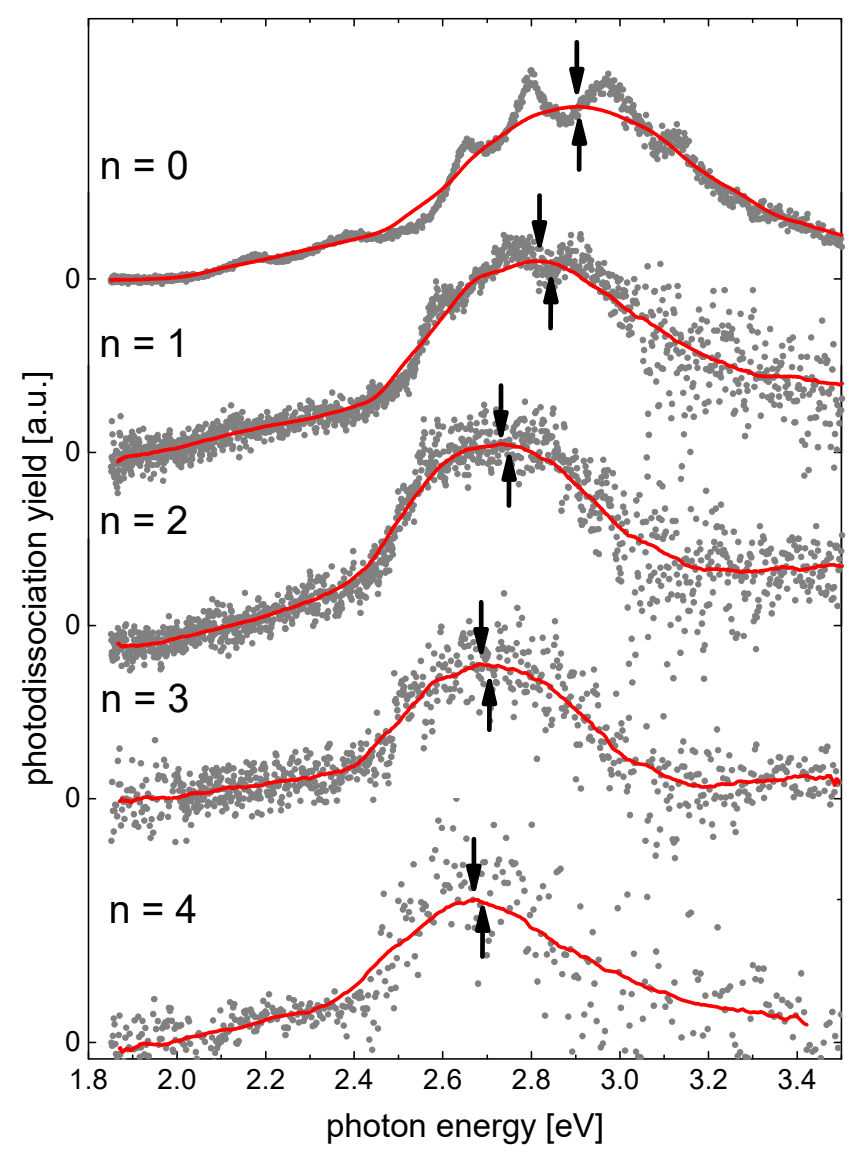

Figure S6. Band envelopes. Experimental data (data points) were smoothed (red curves) with a 200 -point gliding average (100 points for $n=3,4)$. The smoothed curves were interpreted as band envelopes. Arrows above the smoothed curves are the band envelope peaks listed in Table II. Error bars are estimated from small variations in the peak position when using different gliding averages. The MLCT band center of gravity for each spectrum was determined by first integrating the curve underneath the experimental MLCT band. Integration limits were the low-energy end of the experimental data and the point where a 200-point gliding average $(100$ points in case of $n=3,4)$ went through a minimum on the high energy side of the band. The center of gravity is at the energy where the area under the experimental data reaches half the value of the full integral (arrows shown under the smoothed curves, listed in Table II). Uncertainties are estimated from the sensitivity of the procedure to the choice of integration interval. 

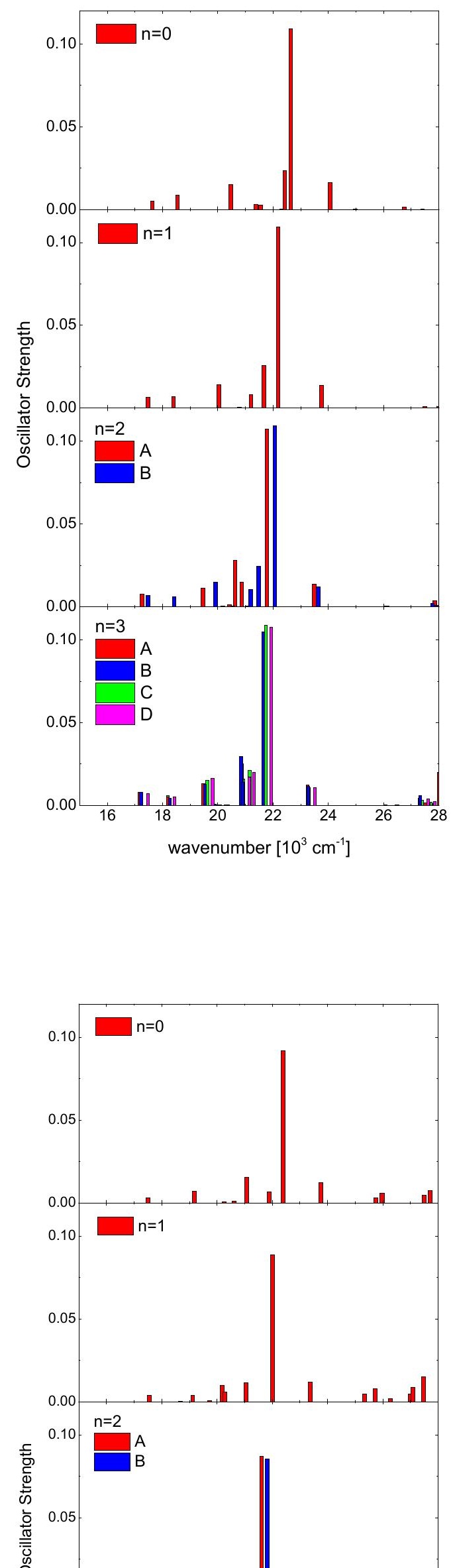
Figure S7. Overview of TDDFT spectra for all isomers. Left: B3LYP/def2-TZVP; right: B3LYP/def2-SV(P). 


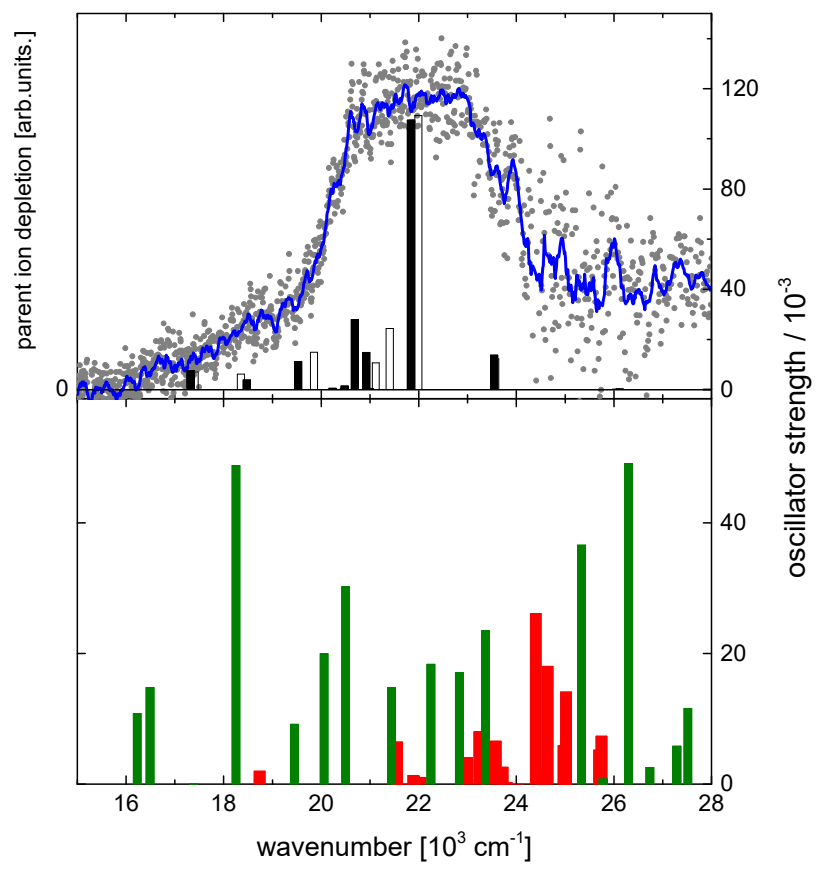

Figure S8. Top panel: Photodepletion spectrum for $\left[\mathrm{RuBT}-\mathrm{OH}_{2}\right]^{2+} \cdot\left(\mathrm{H}_{2} \mathrm{O}\right)_{2}$ and calculated vertical excitation spectrum (columns), shifted by $-1210 \mathrm{~cm}^{-1}(-0.15 \mathrm{eV})$. Circles are raw data points, the full line is a 15-point gliding average. The open columns represent the 2-A isomer, the filled columns show the 2-B isomer. Bottom panel: Calculated vertical excitation spectra of [RuBT$\mathrm{OH}]^{2+}$ (red) and $[\mathrm{RuBT}-\mathrm{OH}]^{+}$(green), shifted by $-1210 \mathrm{~cm}^{-1}(-0.15 \mathrm{eV})$. 